\title{
In Appreciation
}

We thank the following individuals who assessed papers during the year up to 16 November 2011:

\author{
Dr Robert Adams \\ Dr Tony Adiele \\ Dr Vishal Agrawal \\ Dr Taiwo Ajayi \\ Dr Daniel Anderson \\ Dr Ann Arnold \\ Dr Martin Baggaley \\ Dr Benjamin Baig \\ Dr Hadrian Ball \\ Dr James Barrett \\ Dr Anna Beaglehole \\ Professor Paul Bebbington \\ Dr Leo Bergen \\ Dr T. P. Berney \\ Professor Dinesh Bhugra \\ Professor Kamaldeep Bhui \\ Dr Andy Bickle \\ Dr Anne Bird \\ Dr Jed Boardman \\ Dr Jim Bolton \\ Dr Rohan Borschmann \\ Dr Joe Bouch \\ Dr Alexis Bowers \\ Dr Joanna Bredski \\ Dr Andrew Brittlebank \\ Dr Mathew Broome \\ Dr Nicholas Brown \\ Dr Christopher Buckingham \\ Dr Peter Buckley \\ Professor Tom Burns \\ Professor Woody Caan \\ Dr Andrew Cairns \\ Dr Colin Campbell \\ Dr Gavin Cape \\ Professor Carol Caton \\ Dr Pavan Chahl \\ Dr Stephen Chan \\ Dr Mike Chase \\ Dr Arun Chopra \\ Dr Nicola Claxton \\ Dr Martin Commander \\ Dr Mario Jose Francisco Cortina Borja \\ Professor David Cottrell \\ Professor Philip Cowen \\ Professor Nick Craddock \\ Professor Tom K. J. Craig \\ Dr Mike Crawford \\ Dr John Crichton \\ Professor Ilana Crome \\ Dr Sean Cross \\ Dr Martin Curtice \\ Ms Prangya Das \\ Dr Ananta Dave \\ Dr Teifion Davies
}

Dr Steffan Davies

Dr Stephen De Souza

Dr Kimberlie Dean

Dr Arun Dhandayudham

Dr Claire Dibben

Dr Stephen Dinniss

Dr David Dodwell

Dr Nisha Dogra

Dr Anne Doherty

Dr Michael Doyle

Dr Lynne Drummond

Dr Gloria Dura-Vila

Dr Chris Evans

Dr Tim Exworthy

Dr Leonard Fagin

Dr David Fainman

Dr Angela Fan

Dr Mosun Fapohunda

Dr David Fearnley

Dr Larkin Feeney

Professor Phil Fennell

Professor David Foreman

Dr Antony Garelick

Dr Fiona Gaughran

Dr Sanju George

Dr Helen Gosney

Dr Candida Graham

Dr Neil Greenberg

Professor D. Grubin

Dr Allys Guerandel

Professor Gillian Haddock

Dr Rex Haigh

Dr Neel Halder

Dr Michele Hampson

Dr Elizabeth Hare

Professor Keith Hawton

Mrs Rebecca Henry

Dr Isobel Heyman

Professor Fredrick Hickling

Dr Matthew Hodes

Dr Frank Holloway

Professor Louise Howard

Dr Patricia Hughes

Dr Pamela Jacobsen

Dr David James

Dr Michael Jorsh

Dr Renju Joseph

Dr Joseph Kane

Professor Cornelius Katona

Dr Brendan Kelly

Dr Tim Kendall

Dr Naja Khalifa

Dr Joel Khor

Dr Helen Killaspy
Professor Michael King

Professor David Kingdon

Dr Christos Kouimtsidis

Dr Sebastian Kraemer

Dr Matthew Large

Dr Ena Lavelle

Mr Simon Lawton-Smith

Dr Paul Lelliott

Dr Jona Lewin

Dr James Lucey

Dr Seamus Mac Suibhne

Professor Alastair Macdonald

Dr Jonathan Macklin

Professor Anthony Maden

Dr Reuven Magnes

Professor Kevin Malone

Mr Stuart Marchant

Dr Eduard Maron

Dr Susan Mayaki

Mrs Debbie Mayes

Dr Pauline McConville

Dr Hugh Middleton

Dr Gavin Miller

Dr Gabrielle Milner

Dr Willm Mistral

Dr Sally Mitchison

Dr Paul Moran

Dr David Morgan

Dr Jane Morris

Professor Ann Mortimer

Dr Vijaya Murali

Professor David Ndetei

Dr Greg Neate

Professor Jan Neeleman

Dr Giles Newton-Howes

Dr Ramin Nilforooshan

Dr Séamus Ó Ceallaigh

Dr Clare Oakley

Dr Margaret Oates

Dr Colin O'Gara

Dr Denis O'Leary

Professor Femi Oyebode

Dr R. L. Palmer

Dr lan Palmer

Dr Mark Parry

Dr Pradeep Pasupuleti

Dr Fernando Perlado

Dr Nancy Petry

Dr Michael Philpot

Dr Tom Picton

Dr Jonathan Pimm

Dr Vanessa Pinfold

Dr Alexandra Pitman

Dr Adam Polnay 
Dr Norman Poole Professor Rob Poole Dr Stephen Potts Mr Jonathan Radcliffe

Dr Sundararajan Rajagopal

Dr Parashar Ramanuj

Dr Rosalind Ramsay

Dr Gianetta Rands

Dr Gopinath Ranjith

Dr Rahul Rao

Dr Ranga Rao

Dr Lawrence Ratna

Dr Hashim Raza

Dr Paul Reed

Dr Steven Reid

Dr David Reiss

Dr Keith Rix

Professor Graciela Rojas

Dr Nicholas Rose

Dr Robert Rowlands

Dr Gavin Rush

Dr Matthew Sadlier

Dr Sameer P. Sarkar

Dr Jay Sarkar

Dr Ulrike Schmidt

Dr Martin Schmidt

Professor Justine Schneider

Professor Jan Scott

Dr Geoffrey Searle

Dr Neelom Sharma

Dr Viktoria Simon
Professor Swaran Singh

Dr Helen Smith

Dr Luke Solomons

Dr Lucy Spirling

Dr Paul St John-Smith

Dr Nicholas Stafford

Dr Clive Stanton

Dr George Stein

Dr Gary Stevenson

Dr Robert Stewart

Dr Nicholas Stewart

Dr Huw Stone

Dr Anna Streeruwitz

Professor Philip Sugarman

Dr Nicola Swinson

Professor John Swinton

Dr Catherine Symonds

Professor George Szmukler

Professor David Taylor

Dr Mark Taylor

Professor Eric Taylor

Dr Louise Theodosiou

Dr Margaret Thompson

Professor Lindsay Thomson

Dr Gillian Tober

Dr Richard Tranter

Dr Adrian Treloar

Dr Micheline Tremblay

Dr Tongeji Tungaraza

Dr Erin Turner

Professor Peter Tyrer
Dr Stephen Tyrer

Dr Guy Undrill

Dr Rachel Upthegrove

Dr Christopher Vassilas

Dr Akshya Vasudev

Dr David Veale

Dr Andrew Vidgen

Dr Christine Vize

Dr Carsten Vogt

Professor Panos Vostanis

Dr Duncan Waddington

Dr Darryl Wade

Dr Jan Wallcraft

Professor Richard Warner

Dr Tim Weaver

Dr Gavin Wethers

Mr Richard Whale

Dr Oliver White

Dr Alfred White

Dr Simon Wilson

Dr John Wilson

Dr Daniel Wolde-Giorgis

Dr Claire Woolcock

Dr Lisa Wootton

Dr Barry Wright

Professor Graeme Yorston

Professor Allan Young

Professor Wanqing Zhang

Dr Anthony Zigmond

Dr David Zigmond 